\title{
Creating a new kind of healthcare delivery channel- the online mental health marketplace
}

\author{
David J. Lopez, M.D. *
}

University of North Dakota School of Medicine and Health Sciences, Grand Forks, North Dakota, USA

First published online 23 June 2014

In the United States, mental illness affects 1 in 5 Americans. This type of statistic tends to strike close to home. Facilities with individuals suffering with mental illness face the challenge that those who need help should get help, and get good help. Unfortunately, all too often this challenge goes unmet.

One way to address this challenge is to utilize marketbased approaches that successfully leverage the laws of supply and demand and create new healthcare delivery channels for those populations in need of critical mental health services. An innovative market-based approach to address the significant and growing demand for needed mental health services is through the use of Internetbased solutions, or what has traditionally been known as telemedicine.

By utilizing secure Health Insurance Portability and Accountability Act (HIPAA)-compliant audiovideo technology, organizations are beginning to leverage the power of the Internet to deliver real-time mental health services for any number of different groups, regardless of where they are located geographically.

Over the past number of years, the use of a "virtual clinic" business model has been slowly gaining momentum across the country, thereby allowing both government-sector and private-sector enterprises to successfully deliver quality Web-based mental health services to numerous communities throughout the country that otherwise would not have access to these types of services.

The benefits of creating and expanding the presence of these online mental health clinics are many-for both the patients, as well as the providers of these services. They also take advantage of several powerful trends.

These trends include the following:

- Huge and growing demand: An estimated 20 million Americans receive mental health care, accounting for

\footnotetext{
*Address for correspondence, David J. Lopez, MD, Psychiatry Networks, 5158 Broadway Avenue, Cleveland, OH 44127, USA.

(Email: davidjlopez@psychiatrynetworks.com)
}

scores of billions of dollars in fees and medication costs. Recent statistics reveal that approximately 44 million Americans suffer from mental disorders. This is a burgeoning market in the U.S. that accounts for nearly 30 million yearly office visits to physicians for mental disease.

- Limited supply: Large numbers of hospitals and other institutions, along with many rural areas, have no mental health providers at all; there are also not enough psychiatric specialists to meet the needs of large numbers of patients, even in major cities. There are 3711 geographic areas in the U.S. that are designated as Mental Health Professional Shortage Areas by the U.S. Department of Health and Human Services. This trend is worsening due to an increase in patients combined with comparatively low graduation rates for mental health professionals.

- Demand for consumer choice: Many patients are not just willing to receive treatment over the Web; they actually prefer it. Because of the social stigma attached to psychological problems and mental health issues in general, many people seek the privacy (and convenience) offered by Web-based mental health services. This makes treatment more attractive to patients in both urban and remote locations. In fact, going forward, the growth and ease of access of secure online "virtual" mental health clinics may also turn out to generate additional patients who previously resisted counseling, as the use of this new healthcare delivery channel becomes more mainstream and widely accepted.

- Untapped talent: Many mental health professionals have extra capacity outside their normal practices, but no way to serve patients. By eliminating geographical boundaries and time zone limitations, virtual clinics can be set up to allow professionals to generate extra hours and time shift. At the same time that they are able to help more people and generate additional hours and income, they are also able to maintain a good quality of life, as many of these 
providers do not even actually have to leave their own homes or travel anymore in order to deliver mental health services. They are now literally just "a click of the mouse" away from seeing their patients.

Also, while these strong trends are resulting in increased demand, old barriers to telepsychiatry and online mental health services are rapidly disappearing. From widespread broadband video to encryption, the technology to securely, successfully, and effectively deliver these services has arrived, and consumers have grown accustomed to interactions over "the Net".

Just as important, the regulatory environment is catching up. From state medical boards to the U.S. Department of Veterans Affairs (VA), telepsychiatry is now approved (and proven effective by numerous studies). Also, payments are taking off: third-party providers such as insurance companies, academic institutions, and the U.S. government are increasingly paying for telepsychiatry services. Early evidence suggests there will also be direct-to-consumer revenue streams as well, as this new delivery channel evolves and matures.

While the online medical marketplace is still relatively new and growing, given these aforementioned trends, it is very likely that there will be numerous business opportunities in multiple markets for entrepreneurial-minded individuals and organizations with an interest in improving our nation's mental health care system. Telemedicine is one of health care's fastest-growing areas, with many new developments undoubtedly yet to come, particularly in the niche areas of telepsychiatry and online mental health services.

Email: Davidjlopez@psychiatrynetworks.com

\section{Disclosures}

David Lopez, M.D. does not have anything to disclose. 